\title{
Desigualdades sociales en la realización de distintas modalidades de actividad física en Argentina
}

Social inequalities in the patterns of doing different types of physical activity in Argentina

\author{
Matias Salvador Ballesteros \\ Carrera de Sociología e Instituto de Investigaciones Gino Germani de la Universidad de Buenos Aires, CONICET, \\ Argentina \\ matiballesteros@yahoo.com.ar \\ Betina Freidin \\ Carrera de Sociologia e Instituto de Investigaciones Gino Germani de la Universidad de Buenos Aires, CONICET, \\ Argentina
}

\section{Resumen:}

La realización de actividad física se promueve para prevenir enfermedades crónicas no transmisibles y factores de riesgo, y por sus beneficios para el bienestar psico-emocional. Las ciencias sociales y la epidemiología social crítica ponen de relieve los determinantes sociales de distintos tipos de actividades físicas derivados de la clase social, el género y la edad. En este artículo analizamos las intensidades de realización de actividad física global en los distintos grupos sociales, así como las diferencias entre actividades vinculadas al trabajo (doméstico y extra doméstico) y los desplazamientos, y actividades deportivas y/o para mejorar la condición física. En referencia a estas últimas, también describimos el lugar donde se realizan. La fuente de datos utilizada es la Encuesta Nacional de Factores de Riesgo del año 2009, llevada a cabo por el INDEC y el Ministerio de Salud de la Nación con la población urbana de 18 años y más. Este relevamiento, a diferencia de los realizadas en los años 2005 y 2013, permite distinguir entre las actividades físicas vinculadas al trabajo y al desplazamiento de las asociadas a mejorar la condición física y/o hacer deporte. Destacamos la importancia de esta distinción para identificar patrones diferenciales de actividad física de distintos grupos sociales. Palabras ClaVE: Ejercicio, Inequidad Social, Encuestas epidemiológicas.

\section{ABSTRACT:}

Physical activity is encouraged by health experts to prevent chronic non-communicable diseases and risk factors. Physical activity and sports are also beneficial for psycho-emotional well-being. The social sciences and critical social epidemiology point to the social determinants of physical activity and sport, derived from social class, gender and age. In this article we analyze the intensities of global physical activity performed by different social groups, as well as the differences between activities related to work (at home and in the labor market) and commuting, and sports and exercise which are performed to improve or maintain physical fitness. Regarding recreational physical activities, we also describe the place where they are done. Data come from the National Survey of Risk Factors conducted in 2009 with an urban population aged 18 years and over. This survey, unlike those carried out in 2005 and 2013, discriminated between physical activities related to work and commuting and those associated with improving a physical condition and/or playing sports. We emphasize the importance of this differentiation to account for patterns of physical activity performed by different social groups.

KEYwORDs: Physical activity, Social Inequity, Health Surveys.

\section{INTRODUCCIÓN}

La realización de actividad física es parte de los hábitos de vida saludables que son promovidos como prioridades sanitarias de los organismos internacionales, regionales y las autoridades sanitarias nacionales (Organización Mundial de la Salud [OMS], 1986, 2011; Organización Panamericana de la Salud [OPS], 2003, 2012, 2013; Ministerio de Salud [MSAL], 2011, 2015). Sin embargo, las transformaciones sociales, 
económicas, tecnológicas y culturales de las últimas décadas han generado un descenso de las actividades físicas y, por lo tanto, un incremento de los niveles de sedentarismo. La disminución de empleos manuales que requieren un trabajo físico considerable (Ortiz Hernández, 2005), el aumento del parque automotor y una mayor proporción del tiempo libre destinado a actividades sedentarias han colaborado al descenso en la población urbana (OMS, 2002; Barría y Amigo, 2006). En este contexto, la OMS reconoce el papel central de las políticas sanitarias nacionales y de las estrategias intersectoriales para favorecer la actividad física de la población, que incluyen lineamientos de políticas de planificación urbana y de transporte que faciliten el desplazamiento cotidiano, así como la accesibilidad a espacios públicos para el ejercicio recreativo y la práctica deportiva (OMS, 2010).

El término actividad física hace referencia a toda actividad que implica movimiento corporal y gasto energético (Caspersen, Powell \& Christenson, 1985), aunque de acuerdo a la OMS (2002) no existe una definición internacionalmente acordada sobre qué es la actividad física y cómo debe medirse. La OMS (2010, p. 24) distingue diferentes dominios de actividad física para la población adulta activa:

En los adultos de 18 a 64 años, la actividad física se realiza durante el tiempo libre o los desplazamientos (por ejemplo, paseando a pie o en bicicleta) y mediante actividades ocupacionales (es decir, trabajo), tareas domésticas, juegos, deportes o ejercicios programados, en el contexto de las actividades diarias, familiares y comunitarias.

Autores como Ortiz Hernández (2007) agrupan estas actividades y el tiempo implicado en las mismas en dos dominios, el primero refiere al tiempo necesario y las actividades indispensables para la subsistencia y el desempeño de roles sociales (incluyendo aquí el trabajo doméstico y extra doméstico) y el desplazamiento; el segundo refiere al tiempo "libre" que permite la realización personal al destinarse a la sociabilidad y la recreación, incluyendo aquí a la actividad deportiva. En otros trabajos, se distinguen tres dominios de actividad: la ocupacional, la de la vida diaria y la de tiempo libre (Samitz, Egger \& Zwahlen, 2011).

Las encuestas epidemiológicas muestran que la actividad física medida de forma global tiene una fuerte relación inversa con distintas causas de mortalidad, factores de riesgo y enfermedades crónicas no transmisibles (Fleischer, Diez Roux, Alazraqui \& Spinelli, 2008; Samitz et al., 2011). Cuando los cuestionarios incluyen preguntas que diferencien el tipo de actividad (además de la frecuencia, la intensidad y la duración) pueden observarse sus efectos diferenciales para la salud (Cornelio, 2014). A partir de un metanálisis de 80 estudios epidemiológicos realizados en Europa, Norteamérica y Asia, Samitz et al. (2011) concluyen que la actividad física vinculada con el deporte, la vida cotidiana y el tiempo libre tienen una relación inversa con todas las causas de mortalidad, especialmente entre las mujeres; y que esta relación es más intensa que la observada para las actividades físicas vinculadas con el transporte y la ocupación. De hecho, de acuerdo a Cornelio (2014), hay resultados contradictorios respecto de la relación entre la actividad física ocupacional intensa y la salud: algunas investigaciones señalan que está linealmente y otras inversamente relacionada con la mortalidad debido a problemas cardíacos.

Estos estudios nos muestran la importancia de distinguir los dominios y los contextos de la actividad física para analizar su incidencia en la morbi-mortalidad de la población adulta. El análisis sin esta discriminación limita la comprensión de la problemática ya que los distintos tipos de actividades están asociados a diferentes recursos, motivaciones y capacidades de decisión sobre su realización por parte de los sujetos. Por ejemplo, la actividad física ocupacional y la doméstica están relacionadas con la reproducción social, por lo que la decisión sobre su realización excede en muchas ocasiones la voluntad de los sujetos. En cambio, la actividad física vinculada con el deporte y el ejercicio suele realizarse en el tiempo libre y depende de las motivaciones personales y de recursos individuales, interpersonales, comunitarios y residenciales (Ortiz Hernández, 2005; Parra et al., 2010, Diez-Roux \& Mair, 2010; Ballesteros, Freidin, Krause y Borda, 2016). Consultar por los distintos tipos de actividades de forma conjunta puede ocultar el hecho de que determinados grupos sociales realicen en mayor medida un tipo de actividad física y otros grupos realicen otro (Cornelio, 2014; Samitz et al., 2011). Junto con la importancia de discriminar los tipos de actividades entre grupos de diferentes 
edades, y entre varones y mujeres (Samitz et al., 2011), también es necesario hacerlo por la clase social. Por ejemplo, los trabajadores manuales poco calificados pueden ser clasificados en las encuestas epidemiológicas como teniendo una actividad física total semanal intensa, pero al mismo tiempo tener problemas de salud por el exceso y tipo de actividad física que desarrollan en su trabajo diario, y no disponer de tiempo libre ni dinero para realizar actividades físicas recreativas. Bostock (2001) documenta una situación similar para las mujeres de bajos ingresos con hijos pequeños que no poseen auto para desplazarse en su vida diaria y optan por caminar con sus niños. Si bien las caminatas son beneficiosas para la salud, cuando las mujeres lo hacen de manera compulsiva-porque no tienen otra opción de desplazamiento para cortas y medianas distancias - se convierte en una actividad física que aunque por un lado es protectora de la salud, por el otro, genera estrés y fatiga corporal.

De las consideraciones precedentes se deriva la importancia de diferenciar desde la perspectiva de los determinantes sociales de la salud las posibilidades y restricciones para realizar actividad física vinculada con mejorar la condición psicofísica de los sujetos y/o hacer deporte, de los otros tipos de actividades diarias que implican movimiento corporal y gasto energético.

\section{ACTIVIDAD FÍSICA Y DESIGUALDADES SOCIALES: CLASE SOCIAL, GÉNERO Y EDAD}

Las perspectivas sociológicas, la salud colectiva y la epidemiología social crítica enfatizan que las posibilidades de desarrollar estilos de vida saludables en el espacio urbano, incluyendo el ejercicio físico y el deporte, están condicionadas por los modos de vida estructurados por las relaciones de clase, de género y étnicas (Menéndez, 2009). Bourdieu $(1993 ; 2006)$ señala al tiempo libre disponible, al capital cultural y al económico como los principales determinantes de clase para explicar las intensidad y el tipo de deporte realizado, así como el significado y funciones atribuidas a las prácticas corporales. Capriano, Link \& Phelan (2008) y Phelan, Link \& Tehranifar (2010) consideran la posición de clase como la causa fundamental para el acceso desigual a recursos para prevenir enfermedades, controlar factores de riesgo y mitigar los efectos de una condición de salud (por ejemplo, vivir en un barrio seguro y poder pagar una cuota de gimnasio o de un club). El lugar de residencia, a su vez, condiciona el acceso diferencial a infraestructura y a políticas públicas que promueven la realización de actividades recreativas y deportivas (Ballesteros et al., 2016; Ortiz Hernández, 2005; Robledo Martínez, 2006; Hernández, Gómez y Parra, 2010; Parra, et. al., 2010). Las comunidades económicamente más desfavorecidas cuentan con menores recursos colectivos de calidad de vida, como parques y espacios abiertos recreativos (Diez-Roux \& Mair, 2010; Ross, 2011). La desigualdad socio-territorial incluye el mayor riesgo de victimización por episodios de violencia interpersonal en los barrios vulnerabiliza dos de las grandes ciudades y la periferia urbana, afectando el uso de los espacios públicos para actividades recreativas y la sociabilidad (Kilanski y Auyero, 2015; Menéndez, 2009).

El género también produce desigualdades en relación a la actividad física y la práctica deportiva. Estudios internacionales muestran que las mujeres realizan menos deporte y lo hacen con menor regularidad (Tischer, Hartmann-Tews \& Combrink, 2011). El gender gap-aunque ha disminuido en las últimas décadas-se explica por la subordinación en el manejo de recursos económicos, la menor capacidad de disponer de tiempo libre debido al doble rol (económico y reproductivo) y la existencia de una organización sexista del deporte que destina mayor infraestructura y recursos a la práctica deportiva de los hombres. Se suma la violencia simbólica que se ejerce cuando las mujeres practican deportes asociados con roles y valores atribuidos a masculinidad hegemónica (como la competencia, el éxito y la fuerza física) (Hargreaves, 1993; Mosquera González, 2014; Martín, Soler y Vilanova Soler, 2017). Martín et al. (2017) plantean que las desigualdades de género en la práctica deportiva han disminuido en las últimas décadas debido a cambios socioculturales que incluyen una mayor participación de las mujeres en la esfera pública y la relajación de la asociación de la práctica deportiva con los estereotipos de género dominantes. Si bien los estereotipos de género continúan operando y trasmitiéndose de forma más sutil en la vida cotidiana influyendo en los gustos, las elecciones y 
las posibilidades de desarrollar distintas prácticas (Martín, Soler y Vilanova Soler, 2017), Hargreaves (1993: 112) plantea que las configuraciones de poder no son estáticas en la formación cultural que constituyen el deporte y el ocio, y en este sentido, se observan continuidades y discontinuidades culturales a través de procesos de resistencia, acomodación y negociación. Por un lado, las mujeres han desarrollado formas propias de relacionarse con el deporte que no reproducen los valores dominantes de la competencia y el vigor (Martín, Soler y Vilanova Soler, 2017). Pero, por otro lado, junto con los deportes y actividades considerados propiamente femeninos (entre ellos, la gimnasia y el patinaje) también se han desarrollado prácticas que proponen imágenes alternativas de la femineidad con mujeres corriendo maratones, jugando al futbol profesionalmente o haciendo fisicoculturismo, aunque a estas actividades se les otorgue menor visibilidad en los medios masivos (Hargreaves, 1993).En este sentido la autora concluye, "no hay un modelo fijo de opresión masculina en el deporte: algunos hombres y algunas mujeres apoyan los modelos tradicionales, y otros y otras se oponen a ellos" (Hargreaves, 1993, p. 132).

Cabe destacar asimismo que la asociación de la masculinidad hegemónica con el deporte no solo limita la participación de las mujeres en determinadas actividades comparada con su contraparte masculina, sino también la de los adultos mayores en general, en tanto han ido perdiendo los atributos de resistencia, agilidad, coordinación y fortaleza que les demanda el modelo culturalmente dominante (Mosquera González, 2014: 8). Se agrega a ello los impedimentos físicos asociados a una mayor carga de morbilidad (Puig, Barata y Mosquera, 2017). Sin embargo, en las últimas décadas se observa una mayor difusión de la práctica deportiva en el tiempo libre entre grupos de diferentes edades, clases sociales, género y pertenencia étnica en un contexto de diversificación de los lugares para hacerlo y del tipo de deportes (Tischer, Hartmann-Tews y Combrimk, 2001). En el caso de los adultos mayores, se analizan hipótesis alternativas a las del deterioro del estado de salud para explicar su participación en la actividad deportiva atendiendo a las circunstancias sociales (valores culturales y normas sociales) y recursos individuales (tiempo, redes sociales e ingresos) (Tischer, HartmannTews y Combrimk , 2011). También cumplen un rol facilitador para el acceso al ejercicio en el caso de los adultos mayores de menores recursos económicos los programas públicos de promoción del ejercicio y los de la seguridad social.

\section{Metodología Y oBJetivos}

El artículo está basado en el método de análisis de datos cuantitativos secundarios. Como fuente de información se utiliza la Encuesta Nacional de Factores de Riesgo (ENFR) realizada en el año 2009 en Argentina, que ofrece la posibilidad de un análisis empírico de los determinantes sociales de la actividad física. El cuestionario individual contiene una batería de preguntas que permiten clasificar a la población adulta según su nivel de actividad física durante la semana anterior al relevamiento. En esta sección de la encuesta se pregunta por toda la actividad física realizada sin diferenciar el tipo. A diferencia de las ENFR realizadas en el año 2005 y en el año 2013, la ENFR 2009 también incluye preguntas sobre la actividad física realizada durante el último mes, y por los distintos tipos de actividades físicas realizadas en ese período de referencia. En este artículo, a partir de la base de datos de la ENFR 2009, nuestro objetivo es descriptivo. Presentamos un análisis del nivel de actividad física total y del tipo de actividad física, según el nivel educativo, el ingreso del hogar, la pertenencia a un hogar con NBI, el género y el grupo etario de los encuestados. La ENFR no pregunta sobre la ocupación desempeñada por los encuestados, por esta razón consideramos el nivel de educación formal y el ingreso per cápita del hogar como indicadores del nivel socioeconómico. Nos proponemos describir las desigualdades sociales en el sedentarismo, pero también ante los diferentes tipos de actividad física y los lugares en los que se realizan, a la vez que reflexionar metodológicamente sobre las potencialidades y limitaciones que tienen las diferentes formas de medición de la actividad física (total y discriminada por dominios o tipos). 
La ENFR se lleva a cabo de forma conjunta por el Instituto Nacional de Estadísticas y Censos (INDEC) y el Ministerio de Salud desde el año 2005 cada cuatro años. La ENFR 2009 se realizó sobre la base de un diseño probabilístico multietápico (por conglomerados y estratificado), a través de cuatro etapas. Las primeras tres (departamento, área y vivienda) corresponden al marco de muestreo nacional de viviendas, que recortó el universo en localidades urbanas de 5.000 habitantes y más. En total se encuestaron 34.732 personas de 18 años y más, residentes en viviendas particulares (MSAL, 2011). Dado que se trató de un muestreo no proporcional, los realizadores de la encuesta desarrollaron factores de ponderación. En nuestro caso hemos utilizado los factores de ponderación, sin expandir los resultados a toda la población.

En una de las secciones de la ENFR 2009 se le consulta a la población por la realización de actividad física general durante la última semana. Se utilizó una adaptación del IPAQ (International Physical Activity Questionnaire), que indaga diferenciadamente la cantidad de días y minutos por día que durante la última semana se realizó actividad física intensa, moderada y caminata. Se consideró moderada a las actividades que requerían respirar algo más rápido y realizar algún esfuerzo (por ejemplo tareas domésticas, andar en bicicleta lentamente, natación recreativa, etc) e intensas a las actividades que requerían respirar mucho más rápido y realizar un esfuerzo físico mayor (por ejemplo correr, trabajos manuales como construcción, deportes como fútbol o tenis, etc.) (MSAL, 2011). A partir del tiempo y la intensidad de la actividad física realizada durante la última semana se clasifica a la población según su nivel de actividad física en intenso, moderado y bajo.

En una segunda parte de la sección sobre actividad física se indagó sobre la realización de diferentes tipos de actividades físicas en el último mes. Concretamente se pregunta si en el último mes realizó actividad física como actividad doméstica, como parte de su actividad laboral, para desplazarse/trasladarse, y para mejorar su condición física/ hacer deporte. Por último, entre quienes contestaron que realizaron actividad física para mejorar su condición física/ hacer deporte se indagó sobre el lugar de realización y motivaciones.

En este trabajo comenzamos centrando el análisis en la clasificación de la población según su nivel de actividad física (a partir de los criterios de la adaptación del IPAQ). Vemos la diferencia en los porcentajes de población que realiza un nivel de actividad física considerado "bajo" según edad, sexo, nivel educativo, su pertenencia a hogares con Necesidades Básicas Insatisfechas (NBI) y el quintil de ingreso per cápita del hogar en el que reside. En una segunda instancia, a partir de las mismas variables sociodemográficas, examinamos la realización de distintos tipos de actividades físicas en el último mes. Por último, nos centramos en el lugar de realización de la actividad física para mejorar la condición física o por deporte.

Para analizar los datos accedimos a la base de datos, al cuestionario de la encuesta, a la publicación oficial de los resultados y a la guía de usuarios de la base de datos. Esto nos permitió, utilizando el software estadístico SPSS, recodificar los sistemas de categorías originales de algunas variables y realizar cruces de variables diferentes a los publicados por el Ministerio de Salud.

\section{Resultados}

Trabajando con el nivel de actividad física en la última semana, construido con base a la adaptación realizada del cuestionario IPAQ (International Physical Activity Questionnaire), en la Tabla 1 vemos que es más frecuente que la población de menor nivel educativo realice un nivel de actividad física bajo (64,5\%) que los de nivel superior (51,3\%), así como es más frecuente que suceda entre las mujeres $(58,6 \%)$ que entre los hombres (50,9\%), y entre la población mayor de 65 años $(67,0 \%)$ que entre la de 18 a 24 (45,9\%). En cambio, no hay diferencias entre los miembros de los hogares pertenecientes al primer quintil de ingreso per cápita $(53,7 \%)$ y los del quinto quintil $(53,2 \%)$, y es muy débil entre los pertenecientes a hogares con NBI $(52,0 \%)$ y sin NBI $(55,5 \%)$. Es probable que no observemos diferencias en estas variables estructurales debido a que la medición global de niveles de actividad física -sin discriminar por tipo de actividad realizada - lleve a que las actividades semanales entre los distintos grupos sociales puedan "compensarse". Los grupos de ingresos menores y con NBI pueden hacer más actividad física por desempeñar trabajos de tipo manual, 
para trasladarse cotidianamente y por trabajo doméstico; mientras los de ingresos más altos y sin NBI pueden hacer más actividad física recreativa (como veremos más adelante en la Tabla 2, algunas diferencias se hacen visibles al discriminar por tipo de actividad, aunque el período de referencia por el que se consulta ya no es la última semana sino los últimos 30 días). Por otra parte, el nivel educativo puede marcar diferencias en los niveles de intensidad de actividad física medidos a nivel global, por la interacción entre esta variable y la edad (los jóvenes tienen mayores niveles educativos).

Tabla 1. Porcentaje de población que realizó un nivel de actividad física bajo en la última semana y porcentaje de la población que realizó algún tipo de actividad física durante el último mes, según nivel de instrucción, quintil de ingreso per cápita del hogar, sexo, Necesidades Básicas Insatisfechas (NBI) y grupo etario. Población residente en zonas urbanas de 5000 habitantes y más de Argentina en el 2009.

\begin{tabular}{|c|c|c|c|}
\hline & & $\begin{array}{l}\text { Nivel de intensidad } \\
\text { fisica bajo en última } \\
\text { semana }\end{array}$ & $\begin{array}{l}\text { Realizó actividad } \\
\text { fisica en último mes }\end{array}$ \\
\hline \multirow{4}{*}{$\begin{array}{l}\text { Nivel de } \\
\text { instrucción }{ }^{1}\end{array}$} & Hasta primario incompleto & $64,5 \%$ & $64,9 \%$ \\
\hline & $\begin{array}{l}\text { Primario completo y } \\
\text { secundario incompleto }\end{array}$ & $57,0 \%$ & $75,3 \%$ \\
\hline & $\begin{array}{l}\text { Secundario completo y } \\
\text { superior incompleto }\end{array}$ & $51,5 \%$ & $81,5 \%$ \\
\hline & Superior completo & $51,3 \%$ & $85,0 \%$ \\
\hline \multirow{5}{*}{$\begin{array}{l}\text { Quintiles de } \\
\text { ingreso per } \\
\text { cápita del } \\
\text { hogar }^{2}\end{array}$} & 1 & $53,7 \%$ & $77,0 \%$ \\
\hline & 2 & $53,8 \%$ & $77,0 \%$ \\
\hline & 3 & $57,1 \%$ & $73,4 \%$ \\
\hline & 4 & $55,6 \%$ & $78,2 \%$ \\
\hline & 5 & $53,2 \%$ & $82,2 \%$ \\
\hline \multirow{2}{*}{ Sexo } & Varón & $50,9 \%$ & $79,5 \%$ \\
\hline & Mujer & $58,6 \%$ & $76,3 \%$ \\
\hline \multirow{2}{*}{ NBI } & Hogar con NBI & $52,0 \%$ & $77,2 \%$ \\
\hline & Hogar sin NBI & $55,5 \%$ & $77,9 \%$ \\
\hline \multirow{5}{*}{$\begin{array}{l}\text { Grupo } \\
\text { etario }\end{array}$} & 18 a 24 años & $45,9 \%$ & $85,4 \%$ \\
\hline & 25 a 34 años & $49,3 \%$ & $80,8 \%$ \\
\hline & 35 a 49 años & $55,1 \%$ & $78,4 \%$ \\
\hline & 50 a 65 años & $59,5 \%$ & $75,9 \%$ \\
\hline & 66 años y más & $67,0 \%$ & $66,7 \%$ \\
\hline \multirow{3}{*}{ Total } & & $55,0 \%$ & $77,8 \%$ \\
\hline & & 19114 & 27016 \\
\hline & & & $\mathrm{n}=34.732$ \\
\hline
\end{tabular}

Fuente: elaboración propia en base a datos de la ENFR 2009

1 No se incluyó a la población con educación especial, que representaron el 0,1\% de los encuestados.

2 No se incluyó a la población que no respondió a los ingresos totales del hogar, que representaron el 16,8\% de los encuestados.

Una segunda sección del cuestionario permite diferenciar entre quienes realizaron algún tipo de actividad física en el último mes y quienes no realizaron actividad física, más allá de su intensidad. En la Tabla 1 (segunda columna) puede observarse que es más frecuente realizar actividad física durante el último mes entre quienes tienen estudios superiores completos $(85 \%)$ que entre quienes tienen hasta primario incompleto (65\%) y entre el grupo etario de 18 a 24 años (85\%) que el de 66 y más (67\%). A su vez, también hay una relación, aunque más débil, según el género ya que es levemente más frecuente que hayan realizado actividad 
física durante el último mes los hombres (80\%) que las mujeres (76\%). Por último, vemos que en cuanto al quintil de ingreso per cápita del hogar no hay una relación lineal (los que menos actividad física realizan son los del tercer quintil de ingreso) ni hay diferencias entre los miembros de hogares con NBI (77\%) y los miembros de hogares sin NBI (78\%).

Resumiendo los resultados de las variables socioeconómicas analizadas podemos señalar que hasta aquí encontramos diferencias muy débiles entre la presencia de NBI en los hogares y su ingreso con la intensidad de actividad física en la última semana y para la realización de actividad física durante el último mes, mientras que con el nivel educativo fueron más intensas. Entonces, cabe preguntarse si las variables socioeconómicas son importantes para analizar la actividad física de la población. En apariencia, los resultados presentados hasta aquí contradicen parte de nuestro desarrollo teórico. Sin embargo, cuando diferenciamos por tipos de actividad física que realizan los encuestados (Tabla 2), vemos que las diferencias aparecen y nos ayudan a comprender por qué las variables de ingreso y NBI tienen una relación débil con la actividad física global. Como veremos a continuación, mientras que los sectores de menores recursos realizan más actividad física laboral, por traslado y tareas doméstica, los sectores de más recurso realizan más actividad para mejorar la condición física o practicar deporte. Antes de comenzar con el análisis del tipo de actividad que realizan los distintos grupos sociales, cabe destacar que el mismo está centrado solamente en la población que realizó actividad física durante el último mes (que como se observa en la Tabla 1, corresponde al 77,8\% de la población, $\mathrm{n}=27.016$ ).

Con relación a la actividad física doméstica, en la Tabla 2 vemos que es más frecuente realizar actividad física por este motivo entre quienes tienen hasta primario incompleto (52\%) que entre quienes tienen superior completo (43\%), entre los que viven en hogares del primer quintil de ingresos (53\%) que entre los del 5 to quintil (43\%), entre las mujeres (59\%) que entre los hombres (33\%), y entre los miembros de hogares con NBI (52\%) que entre los miembros de hogares sin NBI (46\%). Es decir, son los grupos estructuralmente más desfavorecidos los que cuando realizan actividad física, en mayor proporciónlo hacen debido a actividades domésticas. 
Tabla 2. Porcentaje de población que realizó distintos tipos de actividad física, según nivel de instrucción, quintil de ingreso per cápita del hogar, sexo, Necesidades Básicas Insatisfechas (NBI) y grupo etario. Población residente en zonas urbanas de 5000 habitantes y más de Argentina en el 2009, que realizó alguna actividad física durante el último mes.

\begin{tabular}{|c|c|c|c|c|c|}
\hline & & $\begin{array}{l}\text { Actividad } \\
\text { doméstica }\end{array}$ & $\begin{array}{l}\text { Activida } \\
\text { d laboral }\end{array}$ & $\begin{array}{c}\text { Desplazarse/ } \\
\text { Trasladarse }\end{array}$ & $\begin{array}{c}\text { Mejorar } \\
\text { condición física / } \\
\text { deporte }\end{array}$ \\
\hline \multirow{4}{*}{$\begin{array}{c}\text { Nivel de } \\
\text { instrucción } \\
\end{array}$} & $\begin{array}{l}\text { Hasta primario } \\
\text { incompleto }\end{array}$ & $52,0 \%$ & $34,0 \%$ & $79,1 \%$ & $29,0 \%$ \\
\hline & $\begin{array}{l}\text { Primario completo y } \\
\text { secundario incompleto }\end{array}$ & $49,4 \%$ & $37,4 \%$ & $74,8 \%$ & $41,9 \%$ \\
\hline & $\begin{array}{l}\text { Secundario completo y } \\
\text { superior incompleto }\end{array}$ & $43,7 \%$ & $32,4 \%$ & $70,7 \%$ & $55,8 \%$ \\
\hline & Superior completo & $43,2 \%$ & $31,7 \%$ & $66,2 \%$ & $64,5 \%$ \\
\hline \multirow{5}{*}{$\begin{array}{l}\text { Quintiles } \\
\text { de ingreso } \\
\text { per cápita } \\
\text { del hogar }{ }^{2}\end{array}$} & 1 & $52,9 \%$ & $41,5 \%$ & $80,8 \%$ & $35,1 \%$ \\
\hline & 2 & $48,4 \%$ & $38,7 \%$ & $75,1 \%$ & $43,8 \%$ \\
\hline & 3 & $46,6 \%$ & $35,4 \%$ & $72,3 \%$ & $47,6 \%$ \\
\hline & 4 & $45,0 \%$ & $30,0 \%$ & $68,6 \%$ & $52,8 \%$ \\
\hline & 5 & $43,1 \%$ & $27,7 \%$ & $67,4 \%$ & $64,7 \%$ \\
\hline \multirow{2}{*}{ Sexo } & Varớn & $32,7 \%$ & $42,9 \%$ & $68,7 \%$ & $54,1 \%$ \\
\hline & Mujer & $59,3 \%$ & $26,6 \%$ & $75,6 \%$ & $45,1 \%$ \\
\hline \multirow{2}{*}{ NBI } & Hogar con NBI & $52,4 \%$ & $41,1 \%$ & $80,2 \%$ & $33,0 \%$ \\
\hline & Hogar $\sin \mathrm{NBI}$ & $45,7 \%$ & $33,3 \%$ & $71,1 \%$ & $51,9 \%$ \\
\hline \multirow{5}{*}{$\begin{array}{l}\text { Grupo } \\
\text { etario }\end{array}$} & 18 a 24 años & $39,4 \%$ & $32,2 \%$ & $74,4 \%$ & $57,8 \%$ \\
\hline & 25 a 34 años & $43,8 \%$ & $39,1 \%$ & $72,9 \%$ & $52,6 \%$ \\
\hline & 35 a 49 años & $48,9 \%$ & $41,7 \%$ & $71,3 \%$ & $47,7 \%$ \\
\hline & 50 a 65 años & $48,3 \%$ & $35,7 \%$ & $69,9 \%$ & $45,8 \%$ \\
\hline & 66 años y más & $54,4 \%$ & $13,0 \%$ & $74,1 \%$ & $40,9 \%$ \\
\hline \multirow{2}{*}{\multicolumn{2}{|c|}{ Total }} & $46,6 \%$ & $34,4 \%$ & $72,3 \%$ & $49,4 \%$ \\
\hline & & 12586 & 9282 & 19535 & 13347 \\
\hline
\end{tabular}

Fuente: elaboración propia en base a datos de la ENFR 2009

1 No se incluyó a la población con educación especial, que representaron el 0,1\% de los encuestados.

2 No se incluyó a la población que no respondió a los ingresos totales del hogar, que representaron el 16,8\% de los encuestados.

Con la actividad física laboral sucede algo similar, ya que son las personas pertenecientes a los hogares del primer quintil de ingreso (42\%) y a hogares con NBI (41\%) los que más frecuentemente realizan este tipo de actividad física, mientras que las personas pertenecientes al quinto quintil de ingreso (28\%) y a hogares sin NBI (33\%) son las que menos lo realizan. Sin embargo, con relación al nivel educativo la relación es menos intensa y no es completamente lineal, ya que son las personas con estudios primarios completos y secundarios incompletos (37\%) las que en mayor medida realizan este tipo de actividad, seguidas de las que tienen hasta primario incompleto (34\%), mientras las que menos realizan son las que cuentan con estudios superiores (32\%).Por otro lado, a diferencia de la actividad doméstica, los hombres (43\%) realizan más actividad física laboral que las mujeres (27\%). Entendemos que detrás de esta asociación se encuentra el tipo de ocupación que tienen los sujetos. Las actividades de tipo manual son más frecuentes entre los hombres y las personas de menor nivel educativo, a la vez que están peor remuneradas (por eso es más común que haya más miembros de hogares con bajos ingresos y con NBI). La leve asociación con el nivel educativo está vinculada con el hecho de que entre la población con menor nivel educativo tiene más peso la población de mayor edad que en mayor proporción no se encuentra activa en el mercado laboral y por eso no realizan este tipo de actividad. Como 
puede observarse en la Tabla 3, cuando se controla esa relación por edad, son las personas con menor nivel educativo las que más frecuentemente realizan actividad física laboral y las de mayor nivel educativo las que menos lo realizan, con una diferencia porcentual de 10 puntos o más entre los niveles extremos para todos los grupos etarios (con excepción del grupo de mayor edad, donde la relación se revierte).

TABLA 3

Realización de actividad física durante el último mes para mejorar la condición física o hacer deporte por nivel de instrucción y según grupo etario. Población residente en zonas urbanas de 5000 habitantes y más de Argentina en el 2009, que realizó alguna actividad física durante el último mes.

\begin{tabular}{|c|c|c|c|c|c|c|}
\hline \multirow[b]{2}{*}{$\begin{array}{c}\text { Rango de } \\
\text { edad }\end{array}$} & \multirow[b]{2}{*}{$\begin{array}{c}\text { Actividad } \\
\text { fisica como } \\
\text { parte de } \\
\text { actividad } \\
\text { laboral }\end{array}$} & \multicolumn{4}{|c|}{ Nivel de instrucción } & \multirow[b]{2}{*}{ Total } \\
\hline & & $\begin{array}{c}\text { Hasta } \\
\text { primario } \\
\text { incompleto }\end{array}$ & $\begin{array}{l}\text { Primario } \\
\text { completo y } \\
\text { secundario } \\
\text { incompleto }\end{array}$ & $\begin{array}{l}\text { Secundario } \\
\text { completo y } \\
\text { superior } \\
\text { incompleto }\end{array}$ & $\begin{array}{l}\text { Superior } \\
\text { completo }\end{array}$ & \\
\hline \multirow{3}{*}{$\begin{array}{l}18 \text { a } 24 \\
\text { años }\end{array}$} & $\mathrm{Si}$ & $49,8 \%$ & $37,2 \%$ & $27,7 \%$ & $18,1 \%$ & $32,2 \%$ \\
\hline & No & $50,2 \%$ & $62,8 \%$ & $72,3 \%$ & $81,9 \%$ & $67,8 \%$ \\
\hline & Total & 221 & 2003 & 2714 & 144 & 5082 \\
\hline \multirow{3}{*}{$\begin{array}{l}25 \text { a } 34 \\
\text { años }\end{array}$} & $\mathrm{Si}$ & $45,7 \%$ & $45,4 \%$ & $36,1 \%$ & $35,2 \%$ & $39,2 \%$ \\
\hline & No & $54,3 \%$ & $54,6 \%$ & $63,9 \%$ & $64,8 \%$ & $60,8 \%$ \\
\hline & Total & 219 & 1842 & 2695 & 1152 & 5908 \\
\hline \multirow{3}{*}{$\begin{array}{l}35 \text { a } 49 \\
\text { años }\end{array}$} & $\mathrm{Si}$ & $45,0 \%$ & $47,3 \%$ & $40,0 \%$ & $34,2 \%$ & $41,7 \%$ \\
\hline & No & $55,0 \%$ & $52,7 \%$ & $60,0 \%$ & $65,8 \%$ & $58,3 \%$ \\
\hline & Total & 467 & 2604 & 2241 & 1596 & 6908 \\
\hline \multirow{3}{*}{$\begin{array}{l}50 \text { a } 65 \\
\text { años }\end{array}$} & $\mathrm{Si}$ & $42,6 \%$ & $37,7 \%$ & $31,9 \%$ & $31,7 \%$ & $35,7 \%$ \\
\hline & No & $57,4 \%$ & $62,3 \%$ & $68,1 \%$ & $68,3 \%$ & $64,3 \%$ \\
\hline & & 702 & 2402 & 1444 & 1005 & 5553 \\
\hline \multirow{3}{*}{$\begin{array}{l}66 \text { años y } \\
\text { más }\end{array}$} & $\mathrm{Si}$ & $12,1 \%$ & $12,1 \%$ & $13,9 \%$ & $17,1 \%$ & $13,0 \%$ \\
\hline & No & $87,9 \%$ & $87,9 \%$ & $86,1 \%$ & $82,9 \%$ & $87,0 \%$ \\
\hline & Total & 793 & 1608 & 722 & 415 & 3538 \\
\hline
\end{tabular}

Fuente: elaboración propia en base a datos de la ENFR 2009

En cuanto a la actividad física por traslado, vemos que vuelve a ser más frecuente entre quienes tienen menor nivel educativo ( $79 \%$ frente a $66 \%$ de quienes tienen superior completo), entre las mujeres (76\% frente a $69 \%$ de los hombres) y entre quienes pertenecen a hogares con NBI ( $41 \%$ frente a $33 \%$ )y de menores ingresos per cápita ( $81 \%$ frente a $67 \%$ de los hogares del quinto quintil). Las diferencias entre los distintos grupos etarios es muy débil y la relación no es lineal.

A diferencia de los motivos anteriores para realizar actividad física, es más frecuente la actividad para mejorar su condición física o por deporte entre quienes tienen estudios superiores completos $(65 \%$ frente a $29 \%$ de primaria incompleta), los hombres (54\% frente a $45 \%$ ) y los pertenecientes a los hogares de mayores ingresos per cápita (65\% frente a $35 \%$ del primer quintil) y que no tienen NBI (52\% frente a 33\%). Es decir, es más frecuente realizar actividad física por deporte o para mejorar la condición física entre los grupos estructuralmente favorecidos. Por su parte, la frecuencia de realizar actividad física por este motivo desciende a medida que aumenta el grupo etario (57,8\% entre las personas de 18 a 24 años frente a $40,9 \%$ de quienes tienen más de 65 años).

Otra temática que permite abordar la ENFR 2009 y nos da herramientas para caracterizar la forma en que realizan actividad física distintos grupos sociales, es el lugar de su realización para quienes la realizan por 
deporte o para mejorar su condición física. La Tabla 4 nos muestra que los espacios públicos son el principal lugar, ya que más de la mitad de la población (52,2\%) lo indicó como ámbito de desarrollo de estas prácticas. Por su parte, un poco menos de un quinto señaló a los clubes $(17,4 \%)$ y los gimnasios $(19,4 \%)$, mientras que solo un $10,6 \%$ lo realizó en otros lugares.

Tabla 4. Lugar de realización de actividad física por deporte o para mejorar la condición física según nivel de instrucción, quintil de ingreso per cápita del hogar, sexo, Necesidades Básicas Insatisfechas (NBI) y grupo etario. Población residente en zonas urbanas de 5000 habitantes y más de Argentina en el 2009.

\begin{tabular}{|c|c|c|c|c|c|c|}
\hline & & Club & Gimnasio & Espacio público & Otro $0^{3}$ & Total \\
\hline \multirow{4}{*}{$\begin{array}{l}\text { Nivel de } \\
\text { instrucción }\end{array}$} & Hasta primario incompleto & 7,0 & 8,5 & 75,4 & 9,1 & $(696)$ \\
\hline & $\begin{array}{l}\text { Primario completo y } \\
\text { secundario incompleto }\end{array}$ & 14,8 & 12,9 & 61,5 & 10,7 & $(4382)$ \\
\hline & $\begin{array}{l}\text { Secundario completo y } \\
\text { superior incompleto }\end{array}$ & 20,6 & 22,1 & 47,2 & 10,1 & $(5473)$ \\
\hline & Superior completo & 17,5 & 27,4 & 43,7 & 11,4 & $(2782)$ \\
\hline \multirow{5}{*}{$\begin{array}{l}\text { Quintiles de } \\
\text { ingreso per } \\
\text { cápita del } \\
\text { hogar }\end{array}$} & 1 & 13,1 & 11,1 & 64,5 & 11,4 & $(1691)$ \\
\hline & 2 & 17,0 & 15,9 & 56,6 & 10,7 & $(2000)$ \\
\hline & 3 & 17,0 & 19,0 & 53,9 & 10,1 & $(1950)$ \\
\hline & 4 & 18,0 & 18,9 & 51,9 & 11,3 & $(2150)$ \\
\hline & 5 & 18,8 & 26,0 & 44,6 & 10,6 & $(3166)$ \\
\hline \multirow{2}{*}{ Sexo } & Varón & 25,0 & 16,1 & 48,9 & 10,0 & $(6975)$ \\
\hline & Mujer & 9,0 & 23,1 & 56,7 & 11,2 & $(6372)$ \\
\hline \multirow{2}{*}{ NBI } & Hogar con NBI & 13,1 & 10,9 & 66,9 & 9,1 & (1198) \\
\hline & Hogar sin NBI & 17,8 & 20,3 & 51,2 & 10,7 & (12149) \\
\hline \multirow{5}{*}{$\begin{array}{l}\text { Grupo } \\
\text { etario }\end{array}$} & 18 a 24 años & 22,4 & 23,2 & 42,1 & 12,4 & $(2944)$ \\
\hline & 25 a 34 años & 24,2 & 23,0 & 43,5 & 9,3 & $(3113)$ \\
\hline & 35 a 49 años & 17,5 & 21,1 & 52,8 & 8,6 & $(3298)$ \\
\hline & 50 a 65 años & 8,4 & 14,3 & 66,0 & 11,3 & $(2546)$ \\
\hline & 66 años y más & 8,0 & 9,2 & 69,6 & 13,1 & $(1449)$ \\
\hline \multicolumn{2}{|r|}{ Total } & 17,4 & 19,4 & $\mathbf{5 2 , 6}$ & 10,6 & (13347) \\
\hline
\end{tabular}

Fuente: elaboración propia en base a datos de la ENFR 2009

1 No se incluyó a la población con educación especial, que representaron el 0,1\% de los encuestados.

2 No se incluyó a la población que no respondió a los ingresos totales del hogar, que representaron el 16,8\% de los encuestados. 3 Agrupamos aquí a las categorías Escuela/Universidad, Centro municipal o provincial, Hogar,

Trabajo y Otro. Ninguna de las categorías supera el $4 \%$ de los casos y en su conjunto suman el 10,6\%.

Si bien el patrón observado es a una mayor utilización del espacio público para realizar ejercicio, y en todos los grupos es el principal ámbito para realizar esta práctica, no todos utilizan este espacio en similar proporción. Por el contrario, son los grupos estructuralmente más desfavorecidos quienes en mayor medida, cuando realizan este tipo de actividad física, lo hacen en espacios públicos. Ese lugar es utilizado por el75,4\% de quienes tienen hasta primario incompleto, el 64,5\% de las personas pertenecientes a hogares de menos ingresos, el 66,9\% de quienes viven en hogares con NBI y el $56,7 \%$ de las mujeres, frente al $43,7 \%$ de quienes tienen estudios superiores completos, el 44,6\% de quienes pertenecen a los hogares del quintil de más ingresos, el 51,2\% de quienes residen en hogares sin NBI y el 48,9\% de los hombres. Cabe destacar que esto no significa que los grupos sociales de menores recursos utilicen más el espacio público para realizar actividad física. Como vimos anteriormente, realizan en mucha menor proporción actividad por deporte o para mejorar la condición física que los grupos de mayores recursos, pero cuando lo hacen utilizan en mayor proporción lugares públicos 
que privados. A su vez, el espacio público es más frecuente a medida que aumenta la edad (centrándonos en los extremos, 69,6\% entre la población 65 años y más frente a 42,1\% de quienes tienen 18 a 24 años).

La contracara de lo anterior es que los ámbitos privados, principalmente los gimnasios pero también los clubes, que requieren pagar matriculas y cuotas mensuales, son espacios en donde los sectores de mayores recursos realizan deportes o actividades para mejorar su condición física en mayor proporción que los sectores de menores recursos ${ }^{1}$. Estos establecimientos son los lugares donde en mayor proporción realizan actividades físicas quienes tienen estudios universitarios (17,5\% club y $27,4 \%$ gimnasio) y pertenecen a los hogares del mayor quintil de ingreso per cápita $(18,8 \%$ y $26,0 \%)$ y sin NBI $(17,8 \%$ y $20,3 \%)$; mientras que en menor proporción las realizan allí quienes tienen hasta estudios primarios incompletos (7,0\% y $8,5 \%)$ y pertenecen a los hogares del menor quintil de ingresos per cápita (13,1\% y $11,1 \%)$ y con NBI $(13,1 \%$ y $10,9 \%)$. Cabe destacar una particularidad con relación al género. Mientras es levemente mayor el porcentaje de mujeres $(23,1 \%)$ que de hombres $(16,1 \%)$ que cuando realizan este tipo de actividad física lo hagan en gimnasios; es mucho mayor el porcentaje de hombres $(25,0 \%)$ en comparación con las mujeres $(9,0 \%)$ que asisten a clubes para realizar actividad física. En este sentido, cabe destacar que, por un lado, algunos estudios muestran que existe una distribución desigual en los recursos, y por lo tanto en la oferta y la infraestructura, destinada a deportes masculinos y femeninos en las establecimientos deportivos (Martín et al., 2017). Y, por el otro, que el "uso social" de los clubes presenta especificidades por parte de las mujeres y los varones, especialmente en lo que respecta a la práctica deportiva colectiva (Ballesteros et al., 2016).

\section{Conclusiones}

A partir del análisis de los datos de la ENFR 2009 observamos que las personas de menor nivel educativo, de más edad y las mujeres son quienes realizaron en mayor proporción un nivel de actividad física bajo durante la última semana. En cambio, no encontramos diferencias significativas según quintil de ingreso per cápita del hogar y NBI. A su vez, la ENFR 2009 indagó sobre el tipo de realización de actividad física que las personas realizaron durante el último mes, lo que nos permitió avanzar en la comprensión de la problemática desde una perspectiva de los determinantes sociales, a la vez que señalar algunas consideraciones metodológicas. Vimos que las desigualdades sociales se hacen más intensas (nivel educativo) o aparecen (NBI y quintil de ingreso per cápita del hogar) para la realización de actividades físicas durante el último mes debido a deporte o para mejorar la condición física. En cambio, los resultados de la ENFR 2009 muestran que cuando las personas de grupos estructuralmente desfavorecidos (mujeres, de menores ingresos, de menor nivel educativo y pertenecientes a hogares con NBI) realizan actividad física lo hacen en mayor medida debido a actividades vinculadas con responsabilidades de su vida cotidiana (actividades domésticas, laborales y de traslado) que las personas de grupos estructuralmente favorecidos.

En cuanto a las consideraciones metodológicas, destacamos que los resultados de la ENFR 2009 muestran que al considerarse todos los tipos de actividad física juntos se puede estar ocultando diferencias sociales entre los distintos tipos de actividades físicas que realizan. Así, similares niveles de actividad física durante la última semana entre personas pertenecientes a hogares de distintos estratos sociales (según quintil de ingreso per cápita y NBI) que se obtienen como resultado de la ENFR 2009, oculta las diferencias existentes en el tipo de actividad. Esta información es particularmente importante por dos razones. Por un lado, como señalamos anteriormente, entre los sectores estructuralmente más desfavorecidos es más frecuente la realización de actividad física debido a aspectos obligatorios de su vida cotidiana vinculados con la reproducción social, mientras que entre los sectores más favorecidos es más importante la actividad física en realizada en el tiempo libre, vinculada con el ocio y la recreación. Por otro lado, los distintos tipos de actividades físicas pueden tener diferentes consecuencias para la salud física y el bienestar emocional. El análisis descriptivo que realizamos en este artículo sobre los patrones de actividad física contribuye al debate sobre las desigualdades sociales en salud desde la perspectiva epidemiológica crítica y la salud colectiva, en la medida en que las prácticas 
individuales que son protectoras para la salud integral no pueden entenderse desligadas de los modos de vida de los distintos grupos sociales, que las restringen o facilitan.

Para finalizar, el lugar de realización de actividad física refleja claras diferencias socioeconómicas, siendo los sectores de menores recursos los que utilizan en mayor medida el espacio público para realizarlas. De ahí la importancia de políticas públicas intersectoriales que fortalezcan las oportunidades de realizar actividad física en ambientes promotores de la salud que sean de libre acceso. Estudios internacionales señalan que la inversión en infraestructura pública es el mejor camino para aumentar la práctica de actividad física deportiva y disminuir las desigualdades sociales para hacerlo (García Ferrando y López Goig, 2017; Diez Roux \& Mair, 2010), incluyendo las producidas por las relaciones de género (Martín et al., 2017) y entre distintos grupos etarios (Puig Barata y Mosquera, 2017). Por último, destacamos que los resultados de la ENFR 2009 reflejan en términos relativos la baja utilización de los clubes deportivos por parte de las mujeres en comparación con los hombres. En este sentido, los datos indican la importancia de fomentar en estas entidades la ampliación de recursos e infraestructura que facilite una mayor participación de las mujeres.

\section{Agradecimientos}

El artículo fue realizado en el marco de los Proyectos UBACYT 20020170100024BA "Experiencias de cuidado de la salud en la periferia de Buenos Aires: desigualdad social, territorio y cultura", PICT 2017-1391 "Desigualdad social, cultura y salud en un barrio de la periferia de Buenos Aires" y PRI (FSOC-UBA) "Desigualdades sociales en estilos y modos de vida vinculados con la salud en zonas urbanas de Argentina, 2007-2014”. Además, el artículo fue parcialmente elaborado en el contexto de la Red INCASI, un proyecto europeo que ha recibido fondos del Programa de investigación e innovación Horizonte 2020 de la Unión Europea, bajo la Marie Skłodowska-Curie GA No 691004 y es coordinado por el Dr. Pedro López-Roldán. Este artículo refleja únicamente la opinión de los autores y la agencia no es responsable del uso que pueda hacerse de la información que contiene.

\section{ReFERENCIAS}

Barría, M. y Amigo, H. (2006). Transición nutricional: una revisión del perfil latinoamericano. Archivos Lat. de nutrición, 56(1), 3-11.

Ballesteros, M.S., Freidin, B., Krause, M. y Borda, P. (2016). La práctica de actividad física entre varones y mujeres de clase media en el AMBA: motivaciones, percepción de beneficios y limitaciones para su continuidad. Educación fisica y ciencia, 18(2), 1-20. Recuperado de https://www.efyc.fahce.unlp.edu.ar/article/view/EFyCe009/7893

Bostock, L. (2011).Pathways of disadvantage? Walking as a mode of transport among low-income mothers. Health and Social Care in the Community, 9(1), 11-18. https://doi.org/10.1046/j.1365-2524.2001.00275.x

Bourdieu, P. (1993). Deporte y clase social. En J. Brohm y J. Barbero (Comp) Materiales de Sociología del Deporte. Madrid: Piqueta.

Bourdieu, P. (2006). La distinción. Criterios y bases sociales del gusto. Buenos Aires: Taurus.

Capriano, M., Link, B. \& Phelan, J. (2008). Social Inequality and Health: Future Directions for the Fundamental Cause Explanation. In A. Laureau y D. Conley (comps.) Social Class. How does it Work. Nueva York: Russell Sage Fundation.

Caspersen, C.J., Powell, K.E. \& Christenson, G.M. (1985). Physical activity, exercise, and physical fitness: definitions and distinctions for health-related research. Public Health Rep, 100(2),126-31.

Cornelio, C.I. (2014). Physical activity in the community: correlates of change and overall mortality in two populationbased cohort (Tesis de Doctorado). Universitat Pompeu Fabra, Department of Experimental and Health Sciences, Barcelona, España. Recuperada de http://www.tdx.cat/handle/10803/287275 
Diez-Roux, A. \& Mair, C. (2010). Neighborhoods and health. Annals of the New York Academy of Sciences, 1186(1), 125-145. https://doi.org/10.1111/j.1749-6632.2009.05333.x

Fleischer, N., DiezRoux, A., Alazraqui, M. \& Spinelli, H. (2008). Social Patterning of Chronic Disease Risk Factors in a Latin American City. Journal of Urban Health: Bulletin of the New York Academy of Medicine, 85(6), 923. https://doi.org/10.1007/s11524-008-9319-2

García Ferrando, M. y Llopis Goig, R. (2017). Estructura social de la práctica deportiva. En M. García Ferrando, N. Puig Barata, F. Lagardera Otero (comps.) Sociología del deporte. Madrid: Alianza Editorial.

Hargreaves, J. (1993). Problemas y promesa en el ocio y los deportes femeninos. En J.M. Brohm y J. Barbero (comps.) Materiales de sociología del deporte. Madrid: Ediciones La Piqueta.

Hernández, A., Gómez, L. F. y Parra, D. C. (2010). Ambientes urbanos y actividad física en adultos mayores: Relevancia del tema para América Latina. Rev. salud pública, 12(2), 327-335.

Kilanski, K. \& Auyero, J. (2015). Introduction. In J. Auyero, P. Bourgois y N. Scheper-Hughes (Eds.) Violence at the Urban Margins. Oxford: Oxford University Press.

Martín, M., Soler, S. y Vilanova Soler, A. (2017). Género y deporte. En M. García Ferrando, N. Puig Barata, F. Lagardera Otero (comps.) Sociología del deporte. Madrid: Alianza Editorial.

Menéndez, E. (2009). De sujetos, saberes y estructuras: introducción al enfoque relacional en el estudio de la salud colectiva. Buenos Aires: Lugar.

Ministerio de Salud (2011). Segunda Encuesta Nacional de Factores de Riesgo. Buenos Aires: Ministerio de Salud.

Ministerio de Salud (2015). Tercera Encuesta Nacional de Factores de Riesgo para enfermedades no trasmisibles. Buenos Aires: Ministerio de Salud.

Mosquera González, M. J. (2014). ¿La mujeres no hacen deporte porque no quieren?, ¿Los hombres practican el deporte que quieren?: el género como variable de análisis de la práctica deportiva de las mujeres y de los hombres. En M. J. Mosquera González (Ed.) IV Ciclo de Conferencias Xénero, Actividade Física e Deporte, 2012-2013. La Coruna: Universidade da Coruña. Recuperado de http://ruc.udc.es/dspace/handle/2183/13594.

Organización Mundial de la Salud (1986). Carta de Ottawa para la promoción de la salud. Ottawa: Organización Mundial de la Salud.

Organización Mundial de la Salud (2002). The World Health Report.Reducing Risks, Promoting Healthy Life. Ginebra: Organización Mundial de la Salud.

Organización Mundial de la Salud (2010). Recomendaciones Mundiales sobre actividad fisica para la salud. Ginebra: Organización Mundial de la Salud. Recuperado de http://apps.who.int/iris/bitstream/10665/44441/1/97892 43599977_spa.pdf

Organización Mundial de la Salud (2011). Informe sobre la situación mundial de las enfermedades no transmisibles 2010. Ginebra: Organización Mundial de la Salud.

Organización Panamericana de la Salud (2003). Cuidado innovador para las condiciones crónicas: agenda para el cambio. Washington: Organización Panamericana de la Salud.

Organización Panamericana de la Salud (2012). Estrategia para la prevención y el control de las enfermedades no transmisibles. Washington: Organización Panamericana de la Salud.

Organización Panamericana de la Salud (2013). Plan de acción para la prevención y el control de las enfermedades no transmisibles. Washington: Organización Panamericana de la Salud.

Ortiz Hernández, L. (2005). Disparidad en la disponibilidad de infraestructura para la actividad física deportiva en los municipios de México. Rev Salud PublicaNutr, 6(4).

Ortiz Hernández, L. (2007). La necesidad de un nuevo paradigma en el campo de la alimentación y la nutrición. En E. J. Carillo Soto y E. Guinsberg (Eds), Desafios de Salud Colectiva (pp. 127-152). Buenos Aires: Lugar.

Parra, D. C., McKenzie, T. L., Ribeiro, I. C., Ferreira Hino, A. A., Dreisinger, M., Coniglio, K., ...\& Simoes, E. J. (2010). Assessing physical activity in public parks in Brazil using systematic observation. American journal of public health, 100(8), 1420-1426. https://doi.org/10.2105/AJPH.2009.181230 
Phelan, J.C., Link, B.G. \& Tehranifar, P. (2010). Social Conditions as Fundamental Causes of Health Inequalities; Theory, Evidence, and Policy Implications. Journal of Health and social Behavior, 51(1), 28-40.

Puig Barata, N. y Mosquera, M.J. (2017). Edad y deporte. En M. García Ferrando, N. Puig Barata, F. Lagardera Otero (comps.), Sociologia del deporte. Madrid: Alianza Editorial.

Robledo Martínez, R. (2006). Características Socioculturales de la Actividad Física en Tres Regiones de Colombia. Rev. salud pública, 8(2), 13-27.

Ross, C. (2011). Collective Threat, Trust, and the Sense of Personal Control. Journal of Health and Social Behavior, $52(3), 287-296$.

Samitz, G., Egger, M. \& Zwahlen, M. (2011). Domains of physical activity and all-cause mortality: systematic review and dose-response meta-analysis of cohort studies. International Journal of Epidemiology, 30, 1382-1400. htt ps://doi.org/10.1093/ije/dyr 112

Tischer, U., Hartmann-Tews, I. \& Combrink, C. (2011). Sport participation of the elderly-the role of gender, age, and social class. EurRevAgingPhysAct, 8(2), 83-91. https://doi.org/10.1007/s11556-011-0087-8

\section{Notas}

1 Si bien los datos agregados para el año 2009 muestran este patrón más general de desigualdad social, cabe agregar que existe una oferta diversa de gimnasios y clubes privados, algunos con cuotas que resultan accesibles para sectores de menores recursos. Se destaca asimismo la existencia en algunas jurisdicciones de polideportivos municipales subvencionados por el estado con cuotas mensuales mucho más bajas que la de los clubes y gimnasios privados que disminuyen las barreras financieras en el acceso al deporte. 